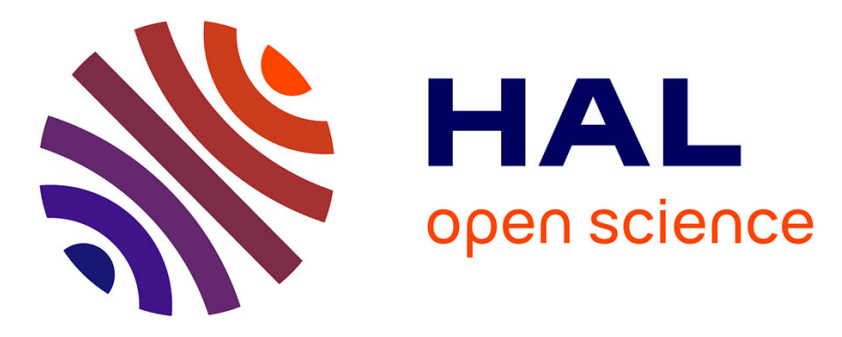

\title{
Ramadan et diabète : est-ce un problème? Considérations pratiques et apport de la mesure continue de la glycémie
}

\author{
L. Monnier, A. El Azrak, D. Rochd, C. Colette, Fabrice Bonnet
}

\section{- To cite this version:}

L. Monnier, A. El Azrak, D. Rochd, C. Colette, Fabrice Bonnet. Ramadan et diabète: est-ce un problème? Considérations pratiques et apport de la mesure continue de la glycémie. Médecine des Maladies Métaboliques, 2015, 9 (6), pp.591-599. 10.1016/S1957-2557(15)30223-6 hal-01243038

\section{HAL Id: hal-01243038 \\ https://hal-univ-rennes1.archives-ouvertes.fr/hal-01243038}

Submitted on 13 Jan 2016

HAL is a multi-disciplinary open access archive for the deposit and dissemination of scientific research documents, whether they are published or not. The documents may come from teaching and research institutions in France or abroad, or from public or private research centers.
L'archive ouverte pluridisciplinaire HAL, est destinée au dépôt et à la diffusion de documents scientifiques de niveau recherche, publiés ou non, émanant des établissements d'enseignement et de recherche français ou étrangers, des laboratoires publics ou privés. 
Ramadan et diabète : Est-ce un problème ?

Considérations pratiques et apport de la mesure continue de la glycémie

Monnier $L^{1}$, El Azrak $A^{2}$, Rochd $D^{2}$, Colette $C^{1}$, Bonnet $F^{3}$

English title : Ramadan and diabetes :Is this of concern ?

Useful considerations and contribution of continuous glucose monitoring

1. Institut Universitaire de Recherche Clinique, Université de Montpellier, Montpellier (France)

2. Groupement de Formation Médicale G11, Casablanca (Maroc)

3. Département d'Endocrinologie, CHU de Rennes, Rennes (France)

Correspondance : Professeur Louis Monnier

Institut Universitaire de Recherche Clinique

641 Avenue du Doyen Giraud

34093 Montpellier Cedex 5, France

e-mail : louis.monnier@inserm.fr 


\section{RÉSUMÉ}

Le mois religieux du Ramadan est caractérisé par l'abstention de toute consommation d'aliments ou de liquides du lever au coucher du soleil. Cette pratique modifie de manière considérable les habitudes alimentaires. Le repas de rupture du jeûne (Iftar) souvent riche en glucides rapides entraine chez les patients diabétiques une brusque montée de la glycémie, qui peut conduire à une hyperglycémie soutenue et chronique sur l'ensemble de la période nocturne, si le repas de rupture du jeûne est suivi soit par un dîner consommé 2 à 3 heures après, soit par plusieurs collations, soit par les deux à la fois. Si on inclut le repas pris avant l'aube (Sohour), la consommation cumulée de glucides sur la période nocturne peut atteindre les 500 grammes. Dans ces conditions, il n'est pas étonnant que l'équilibre glycémique des patients diabétiques, en particulier de ceux qui sont insulinés, soit profondément perturbé pendant le Ramadan. Chez de nombreux patients, l'excès d'apports calorique et glucidique conduit à une prise pondérale. Les évaluations nutritionnelles faites à partir des achats alimentaires réalisés par les ménagères confirment que la consommation calorique est excessive.Compte tenu de ces observations, il apparaît quel la gestion des mesures diététiques et au delà du traitement antidiabétique médicamenteux, en particulier de l'insulinothérapie, n'est pas un problème simple au cours du Ramadan. Dès lors, la meilleure attitude serait d'essayer de déconseiller la pratique du Ramadan chez tous les patients diabétiques quand les conditions suivantes ne sont pas strictement remplies : observance suffisante d'un programme nutritionnel structuré, acceptation et compréhension d'un schéma thérapeutique personnalisé mais codifié. Une autosurveillance glycémique intensifiée devrait au minimum être préconisée chez tous les patients dont l'état de santé nécessite un traitement insulinique par multi-injections. Chez les patients qui n'observent pas ces recommandations, le danger est, dans l'immédiat, la survenue d'évènements aigus (hyperglycémies ou hypoglycémies sévères) au cours du Ramadan, et à plus long terme, l'apparition ou la progression de complications chroniques en raison de « l'effet mémoire » d'une exposition excessive au glucose. En effet, même si cette dernière reste limitée à un mois par an, elle peut se révéler délétère par sa répétition annuelle sur plusieurs années.

Mots clés : Diabète, Ramadan, profils glycémiques, problèmes diététiques et pharmacologiques 
The holy month of Ramadan is characterized by abstinence from eating and drinking from dawn to sunset. This dietary practice entails profound changes in life style habits. The sunset meal (Iftar) that breaks the fasting state is usually followed by exaggerated surges in blood glucose, which can further result in overnight sustained hyperglycaemia in case of nocturnal overfeeding. The latter is usually related to additional food intakes that can be provided as either a dinner taken 2 or 3 hours after the sunset meal or intermittent repeated snacks, and sometimes as a combination of both of them. Throughout the nocturnal period, the cumulative consumption of carbohydrates including the predawn meal (Sohour) can reach a level as high as $\mathbf{5 0 0}$ grams. In such situations, it is not surprising that the glycaemic control of persons with diabetes, especially of those treated with insulin, shows marked disturbances during Ramadan. In many patients, the exaggerated loads in calories and carbohydrates result in body weight gain.Analyses of marketed food supplies confirm this overconsumption and thus this overfeeding. Consequently, both dietary measures and pharmacological glucose-lowering treatments are not easy to handle during Ramadan. The most appropriate attitude would be to convincing persons with diabetes for being exempted from religious fasting when the following conditions are not fulfilled : compliance to a nutritional program and acceptance / understanding of a structured and personalized therapeutic regimen. Assignment to a tight self-monitoring of blood glucose should be recommended at least in those requiring multiple daily injections of insulin. Patients who do not comply to such requirements are at risk for acute adverse events (severe hyperglycaemia and /or hypoglycaemia) during the Ramadan period. In addition, there arises the question as to whether a one-month excess in glucose exposure every year for several decades has or not the potency to exert a deleterious « legacy impact » on the long-term development and/or progression of chronic diabetic complications.

Key words : Diabetes, Ramadan, glycaemic patterns, dietary and pharmacological concerns 
Sur les 7 milliards d'habitants qui peuplent désormais notre planète, le diabète touche environ 350 millions d'entre eux, soit approximativement $5 \%$ de la population mondiale [1]. Les musulmans étant plus de 1,6 milliards à l'échelle mondiale et la proportion de diabétiques étant supposée être la même que dans les autres populations $(5 \%)$, on peut considérer que 80 millions de musulmans sont concernés par cette maladie. En extrapolant les résultats de l'étude Epidemiology of Diabetes and Ramadan (EPIDIAR) [2] et en considérant que 70 à $80 \%$ des musulmans diabétiques pratiquent le jeûne religieux, c'est plus de 50 millions de personnes diabétiques [3] qui sont amenées tous les ans et sur un mois à modifier leur mode de vie et à adapter leurs traitements antidiabétiques à ces changements. Ces données sont probablement sous-estimées, car depuis de nombreuses années, le nombre de personnes atteintes de diabète ne cesse d'augmenter, en particulier dans les pays qui viennent d'accéder à un niveau économique suffisant pour voir apparaître les 2 grands facteurs de l' "épidémie du diabète »: la suralimentation et la sédentarité [4]. Bien que toutes les religions recommandent à leurs fidèles de respecter des périodes de jeûne [5],c'est probablement la religion musulmane qui prône les mesures les plus strictes et les plus longues avec 1 mois de jeûne religieux par an et l'obligation d'éviter toute prise d'aliments et de boissons entre le lever et le coucher du soleil. Chez les personnes en bonne santé, exemptes de toute affection chronique, il n'a jamais été prouvé que le jeûne intermittent de quelques heures exerce des effets néfastes sur la santé. Au contraire, il pourrait avoir des effets bénéfiques sur certains facteurs de risque comme les dyslipidémies par exemple [6]. Pour certaines personnes en surcharge pondérale, il offrirait même la possibilité de perdre quelques kilogrammes [7] pour peu que cet amaigrissement ne soit pas annihilé par un rattrapage pondéral dans la période post Ramadan. II n'en reste pas moins que le Ramadan s'accompagne de modifications notables dans les habitudes alimentaires: rythme et composition des repas. Dans certaines affections chroniques comme le diabète où l'on préconise des prises alimentaires à horaires réguliers avec une charge glucidique relativement stable [8], la rupture de cette régularité risque d'avoir des conséquences délétères sur l'équilibre glycémique, en particulier lorsque les patients sont sous insulinothérapie. Parmi les problèmes soulevés par le jeûne religieux chez les patients diabétiques, le premier est lié à sa durée, qui s'étale sur près d'un mois avec une longueur quotidienne de jeûne diurne qui peut aller de 11 à 18 heures en fonction de la saison et de la zone géographique. 
Un autre problème est lié aux conséquences métaboliques engendrées par le jeûne dès que cet état se prolonge, avec mobilisation des acides gras et production de corps cétoniques [9]. Chez le diabétique de type 2, ces deux phénomènes vont aggraver l'insulinorésistance [10]. Chez les diabétiques de type 1 mal équilibrés, ces perturbations métaboliques peuvent précipiter l'évolution vers une décompensation cétoacidosique.

Compte tenu de tous ces éléments, plusieurs questions méritent d'être posées :

- Le jeûne du Ramadan s'accompagne-t-il de perturbations de l'équilibre glycémique susceptibles de retentir à long terme sur l'état de santé des personnes diabétiques qui le pratiquent?

- Le jeûne du Ramadan s'accompagne-t-il de modifications pondérales ? Le jeûne diurne devrait normalement faire perdre du poids, mais rien n'est moins sûr dans la mesure où sa rupture au cours de la nuit peut donner lieu au minimum à un rattrapage, voire même à une surconsommation calorique.

- Le jeûne du Ramadan devrait-il être déconseillé chez les diabétiques? Si on l'autorise, peut-on le gérer au mieux pour que le déséquilibre glycémique reste dans des limites raisonnables sans conséquences sur le long terme?

Dans tous les cas, la permission ou l'exemption de pratiquer le Ramadan chez un patient diabétique devrait être argumentée à partir du contexte individuel même si des recommandations générales peuvent être formulées. A cet égard, l'abstinence de toute prise de boissons pendant la journée peut être responsable d'états de déshydratation en particulier lorsque le Ramadan est pratiqué dans des zones géographiques très chaudes ou lorsque sa date, fixée à partir du calendrier musulman, coïncide avec une saison chaude. Par ailleurs, il convient de noter que les femmes diabétiques enceintes devraient être dispensées du jeûne religieux car elles constituent un groupe à très haut risque de morbidité et mortalité pour elles mêmes et pour l'enfant qu'elles portent [3].

En 1995, une première conférence regroupant des médecins et des chercheurs s'était tenue à Casablanca pour faire des recommandations sur les aspects psychologiques et physiologiques du Ramadan [11]. Les conclusions furent relativement évasives, et conduisirent les experts à énoncer qu'un patient ayant un diabète de type 2 stable, sans comorbidité évolutive associée et sous traitement par antidiabétiques oraux, peut suivre le Ramadan en toute sécurité. 
En revanche, les autres cas de figures restèrent ouverts à la discussion.

Le but de cette revue est de proposer une actualisation des recommandations à la lumière des nouvelles acquisitions scientifiques fournies, en particulier, par les enregistrements glycémiques continus.

\section{LEÇONS FOURNIES PAR LES ETUDES EPIDEMIOLOGIQUES ET OBSERVATIONNELLES}

\section{Risque d'accidents aigus}

\section{Hypoglycémies}

De manière générale, le jeûne du Ramadan accroît la fréquence des hypoglycémies chez les patients diabétiques.L'étude EPIDIAR [2] a montré que le risque d'hypoglycémie sévère (définie par la nécessité d'une hospitalisation) est multipliée par 4,7 chez les patients ayant un diabète de type 1 (passage de 3 à 14 évènements/100 personnes/mois) et par 7,5 chez ceux qui ont un diabète de type 2 (passage de 0,4 à 3 évènements/100 personnes/mois). II convient de noter que cette augmentation du risque est peut-être sous-estimée car ne furent prises en considération que les hypoglycémies sévères nécessitant une hospitalisation. Celles qui avaient été traitées à domicile grâce à l'assistance d'une tierce personne n'avaient pas été comptabilisées.

Dans une étude récente [12], qui a porté sur 56 patients diabétiques (50 de type 2 et 6 de type 1), il a été observé grâce à un enregistrement glycémique continu que le pourcentage de temps passé en hypoglycémie (glycémie $<3,9 \mathrm{mmol} / \mathrm{l}$ soit $0,70 \mathrm{~g} / \mathrm{L}$ ) n'est pas différent pendant les périodes de Ramadan $(2,4 \pm 1,6 \%)$ et hors Ramadan $(1,1 \pm 1, \%)$. De plus, la variabilité glycémique (Mean Amplitude of Glycemic Excursions, MAGE), qui est un facteur de risque potentiel d'hypoglycémie quand elle est augmentée [13], n'a pas subi d'augmentation significative pendant le Ramadan [12]. 


\section{Hyperglycémies aiguës}

L'étude EPIDIAR [2] a montré que les épisodes d'hyperglycémie sévère, nécessitant une hospitalisation, sont 5 fois plus fréquents chez les diabétiques de type 2 pendant le Ramadan qu'en dehors ( 5 évènements/100 patients/mois vs 1/100 patients/mois). Chez les diabétiques de type 1, l'incidence des épisodes d'hyperglycémie sévère avec ou sans cétoacidose passe de 5 à 17 évènements/100 patients/mois, soit une multiplication par un facteur supérieur à 3. La cause de ces épisodes de décompensation semble dépendre de plusieurs facteurs. Le premier est une réduction excessive et non justifiée des doses d'insuline et/ou d'antidiabétiques oraux. Le deuxième facteur est une non adhérence aux consignes diététiques, caractérisée en général par une augmentation des apports en calories et en sucres rapides $[3,14,15]$.

\section{La dysglycémie chronique du Ramadan}

En dehors des accidents aigus, qui sont en relation avec une inadéquation majeure entre doses d'antidiabétiques et prises alimentaires, la question est de savoir si le Ramadan induit un déséquilibre glycémique soutenu et chronique (hyperglycémie ambiante). A cet égard, il convient de rappeler que le Ramadan dure un mois et que toute exposition excessive au glucose, pour peu qu'elle s'étale sur quelques semaines, entraine :

- Une glycation anormale de l'hémoglobine avec augmentation de l'HbA1c [16]

- Une glycation excessive des protéines de structure des parois vasculaires $[17,18]$ avec un « effet mémoire » qui peut avoir un retentissement plusieurs années après [19].

Ainsi, une période d'exposition anormale à l'hyperglycémie, de 1 mois mais annuelle, correspond sur 24 ans à une hyperglycémie soutenue et cumulée de 24 mois. Nul ne peut affirmer que cette hyperglycémie est anodine. II est même hautement probable qu'elle puisse exercer des effets délétères. La publication récente de Lessan et al [12] mérite une attention particulière. 
Chez des diabétiques explorés par enregistrement glycémique continu sur une période minimum de 2 jours consécutifs, en période de Ramadan et en dehors, ces auteurs ont été conduits à 2 types d'observations intéressantes à maints égards :

- Les durées de temps passées en hyperglycémie et euglycémie sont identiques au cours et en dehors du Ramadan. Toutefois, l' $\mathrm{HbA}_{1 \mathrm{c}}$ n'a pas été mesurée pendant le Ramadan mais uniquement avant $(7,2 \% \pm 1,2 \%)$, probablement parce que ce dosage intègre une période de 3 mois [16] largement supérieure à la durée du Ramadan.

- La rupture du jeûne (Iftar) après le coucher du soleil s'accompagne d'un incrément glycémique franc et aigu de l'ordre de $1 \mathrm{~g} / \mathrm{L}(5 \mathrm{mmol} / \mathrm{l})$ (figures 1 et 2). Cette hyperglycémie persiste pendant toute la nuit, en particulier chez ceux qui sont sous insuline. Le repas pris avant le lever du soleil (Sohour) contribue à réactiver cette hyperglycémie. La glycémie ne retrouve son niveau basal (subnormal dans le groupe entier mais élevé dans le groupe insuline) qu'en fin d'après midi après une décroissance progressive mais lente pendant la période diurne d'abstinence alimentaire (figures 1 et 2).

Les auteurs [12] furent amenés à conclure que le Ramadan n'entraine ni détérioration ni amélioration de l'équilibre glycémique chez des patients diabétiques bien contrôlés à l'état de base et nous ajouterons bien surveillés et conseillés pendant la période du Ramadan.

Cette vision un peu idyllique n'est peut-être pas la plus fréquente. L'augmentation très significative des hospitalisations pour hypoglycémies et hyperglycémies sévères rapportée dans l'étude EPIDIAR [2] est là pour nous rappeler que le Ramadan est une période qui peut s'avérer délicate pour l'équilibre glycémique chez des patients insuffisamment équilibrés en dehors du Ramadan, ne bénéficiant pas d'un encadrement médical suffisant ou se sentant peu concernés par les recommandations de leur médecin, même lorsque l'encadrement médical est présent. Cette opinion semble confortée par l'observation clinique que nous avons rapportée dans un éditorial récent [20]. Chez un patient diabétique de type 2 , traité par une association insuline - antidiabétiques oraux, nous avons eu l'opportunité de réaliser un enregistrement glycémique continu pendant le Ramadan (figure 3). 
Son profil glycémique est voisin de ceux qui ont été rapportés par Lessan et al [12] mais avec une exagération considérable de la montée glycémique au moment de la rupture du jeûne, avec une hyperglycémie soutenue qui s'est maintenue en plateau à $4 \mathrm{~g} / \mathrm{L}$ pendant toute la nuit jusqu'au matin et avec un retour très lent et linéaire pour atteindre un taux subnormal $(1,30 \mathrm{~g} / \mathrm{L})$ en fin d'après midi. Ce patient était traité par une injection d'insuline avant le repas de rupture du jeûne. Cette injection associait de la glargine U100 (44 unités) et de la glulisine (16 unités). De plus, il recevait un traitement oral par metformine (2 $\mathrm{g} / \mathrm{jour}$ ) et glibenclamide (10 mg/jour). Cette observation montre clairement que le jeûne du Ramadan peut s'accompagner de perturbations glycémiques majeures quand les 2 conditions suivantes ne sont pas remplies : équilibre glycémique correct à l'état de base et respect d'un programme nutritionnel et pharmacologique structuré pendant le Ramadan. De plus, l'observation que nous venons de relater soulève 2 problèmes majeurs : a) le retour très lent vers une normoglycémie au cours de la période de jeûne diurne, lorsque la glycémie du matin est très élevée et b) la détérioration considérable de la glycémie pendant la nuit avec une montée glycémique très rapide et très prononcée au moment de la rupture du jeûne. Le premier problème impose un retour vers la physiopathologie et le deuxième un commentaire sur l'observance des mesures diététiques.

\section{RETOUR VERS LA PHYSIOPATHOLOGIE}

Chez les sujets normaux en bonne santé, la capacité d'utilisation du glucose pendant l'état postabsorptif, c'est à dire pendant les 6 heures qui suivent la période postprandiale (4 heures) [21,22] est de l'ordre de 2 mg/kg/min [10]. Pour un individu de $96 \mathrm{~kg}$ (ce qui est le cas du patient dont nous avons rapporté l'observation), ceci correspond à une utilisation horaire du glucose de l'ordre de 12 grammes. Dans la mesure où ce patient a pris un repas (Sohour) avant l'aube, on peut considérer qu'il a été en période postabsorptive entre 11 heures du matin et 17 heures dans l'après midi, c'est à dire pendant une période où la glycémie a chuté de $1 \mathrm{~g} / \mathrm{l}$ en passant de $2,5 \mathrm{~g} / \mathrm{L}$ vers 11 heures à 1,5 g/L vers 17 heures (figure 3 ). Considérant que le volume de distribution extracellulaire du glucose (glucose échangeable) est de $150 \mathrm{ml} / \mathrm{kg}$ de poids corporel [23], la quantité totale de glucose présente dans le pool échangeable de ce patient de 96 $\mathrm{kg}$ à 11 heures du matin (glycémie à $2,5 \mathrm{~g} / \mathrm{L}$ ) pouvait être estimée à 36 grammes. 
La normalisation de la glycémie à $1 \mathrm{~g} / \mathrm{L}$, avec obtention d'une quantité de glucose échangeable normale (14 grammes), soit un décrément de 22 grammes (36 -14 grammes) aurait dû être atteinte en moins de 2 heures si l'utilisation du glucose avait été normale chez ce patient (12 grammes de glucose par heure). En fait, ce n'est qu'en fin d'après midi, aux alentours de 20 heures (figure 3) qu'un taux voisin de 1,30 g/L a pu être atteint. Ceci indique clairement que ce sujet, malgré l'absence de toute prise alimentaire glucidique au cours de la journée, est resté en insulinopénie relative pendant toute la période postabsorptive, avec incapacité d'utiliser le glucose à vitesse normale pendant cette période. En d'autres termes, l'injection d'insuline pratiquée la veille, avant le repas de rupture du jeûne (Iftar) s'est avérée insuffisante pour couvrir les besoins insuliniques diurnes, malgré l'administration, la veille à 20 heures, d'une dose d'insuline relativement élevée (60 unités soit approximativement 0,6 unité/kg de poids/jour). Ainsi il apparaît évident que l'administration d'une dose d'insuline au moment du repas pris avant l'aube (Sohour) aurait été souhaitable voire indispensable pour accélérer la chute de la glycémie pendant la période diurne.

\section{LES APPORTS NUTRITIONNELS SONT-ILS UN PROBLEME MAJEUR PENDANT LE RAMADAN ?}

Les montées glycémiques aiguës observées au moment de la rupture du jeûne (Iftar) chez les diabétiques et le maintien d'une glycémie élevée pendant la nuit $[12,20]$ soulèvent le problème des apports nutritionnels chez les diabétiques pendant le Ramadan. Rappelons brièvement que la réalimentation entre le coucher et le lever du soleil au cours du Ramadan est jalonnée par 2 repas [24]. Le premier, dit de rupture du jeûne (Iftar), suit le coucher du soleil. Le deuxième, dit repas avant l'aube (Sohour), est sensé permettre la couverture des besoins hydriques, énergétiques et glucidiques pour le reste de la journée. 
Le repas de rupture du jeûne à lui seul semble assurer un apport calorique et glucidique raisonnable (1400 kcal et $156 \mathrm{~g}$ de glucides), même si le tiers des glucides (46 grammes) est apporté sous forme de sucres simples [12] fournis en partie par des dattes. En dépit de leur teneur assez forte en fibres (2,5 grammes de fibres brutes pour 100 grammes), les dattes sont surtout riches en sucres simples (68 grammes pour 100 grammes avec une teneur sensiblement égale en glucose et en fructose [25,26]). Dans ces conditions, chez les diabétiques, il ne faut pas être surpris par la montée de la glycémie, même si le pouvoir hyperglycémiant des dattes est en partie « tamponné » par la consommation simultanée de produits laitiers à faible index glycémique.

Ainsi, le repas de rupture du jeûne par lui même ne serait qu'une entorse mineure à la diététique habituelle du diabétique s'il n'était pas suivi par d'autres repas ou collations [24]. Le plus habituel est le dîner qui, 2 à 3 heures plus tard, suit le repas de rupture du jeûne. Couplé à un grignotage nocturne, il contribue à créer une surconsommation calorique et glucidique pendant le mois du Ramadan et à entretenir une hyperglycémie soutenue pendant la période nocturne. Cet excès nutritionnel est difficile à évaluer, mais 2 constatations permettent de penser qu'il est réel. Tout d'abord, plusieurs publications récentes ont montré qu'à l'amaigrissement classique se substitue de plus en plus un gain pondéral au cours du Ramadan $[14,15]$. Ainsi, la prise de poids chez des Egyptiens exempts de toute pathologie a été en moyenne de 1,7 kg chez les hommes et de 2,6 kg chez les femmes [14]. L'évaluation nutritionnelle réalisée dans cette même étude [14] a montré que le Ramadan conduit à des augmentations sur l'apport calorique total (de l'ordre de 200 à $250 \mathrm{kcal} / \mathrm{jour}$ ), sur les apports lipidiques (de l'ordre de plus de $4 \%$ de l'énergie totale), en sucre (20 à $30 \mathrm{~g} / \mathrm{jour}$ ) et en produits laitiers (environ $20 \mathrm{~g} / \mathrm{jour}$ ). Des résultats superposables ont été rapportés en Arabie Saoudite [15] :prise de poids chez $60 \%$ des sujets, augmentation de la consommation de produits alimentaires gras ou sucrés à la rupture du jeûne et de riz au repas pris avant l'aube. Les causes de cette surconsommation sont attribuées par ordre décroissant à des facteurs sociaux (invitations, célébrations), psychologiques (« désir d'inviter les autres ») et religieux (« scilate arrahime », visite de proches parents). Les enquêtes d'achat alimentaire à partir du panier de la ménagère confirment cette surconsommation, même s'il est toujours hasardeux d'extrapoler les quantités alimentaires achetées à celles qui sont réellement consommées. 
Une enquête réalisée au Maroc en 2006-2007 montre que les dépenses alimentaires augmentent de 18,6\% pendant le Ramadan, avec une mention particulière pour les fruits frais et secs $(+104,1 \%)$, les produits laitiers $(+62,2 \%)$, les boissons $(+53,8 \%)$, les viandes et les volailles $(+22,9 \%)$. Des observations identiques ont été faites en Tunisie par l'Institut National de la Consommation. Les quantités consommées (Ramadan versus hors Ramadan) exprimées par personne et par mois sont les suivantes : lait 2 litres vs 0,9 ; yaourts 12,9 pots vs 5,4 ; œufs 26 unités vs 12,8 ; baguette de pain $1,4 \mathrm{~kg}$ vs 0,6 ; viande $3,24 \mathrm{~kg}$ vs 2,25 . Dans ces conditions, un gain pondéral de l'ordre de $2 \mathrm{~kg}$ sur une période d'un mois n'a rien de surprenant. Quand on sait qu'une prise de poids de $2 \mathrm{~kg}$ correspond à un gain énergétique de l'ordre de $14400 \mathrm{kcal}$ [27], on peut en déduire que l'augmentation des apports caloriques quotidiens se situe aux alentours de $14400 \mathrm{kcal} / 30=480 \mathrm{kcal}$. La répartition de cet excès d'apport calorique au cours de la période nocturne est difficile à préciser car les habitudes alimentaires varient d'un pays à l'autre, d'une famille à l'autre, mais également d'une saison à l'autre. Au Maroc, il semblerait que ce soit le repas de rupture du jeûne et le dîner à suivre qui représentent la majorité de l'apport calorique, comme l'illustre l'assortiment de plats marocains présentés sur la figure 4. Dans la péninsule arabique, c'est le repas avant l'aube qui serait le plus riche. Sur la figure 5 , nous avons tenté de schématiser la répartition des glucides sur l'ensemble de la nuit : 150 grammes au repas de rupture du jeûne, 80 grammes au repas avant l'aube avec des quantités difficiles à déterminer pour le dîner qui suit le repas de rupture du jeûne et pour les collations nocturnes. Ces quantités, qui peuvent aller jusqu'à 300 grammes de glucides, peuvent transformer un apport glucidique normal $(150+80=230$ grammes/jour $)$ en apport excessif de l'ordre de $230+300$ soit 530 grammes par jour. Même si l'augmentation moyenne des apports glucidiques se situe probablement entre 50 et 100 grammes/jour, il est facile de comprendre pourquoi certains diabétiques grossissent pendant le Ramadan et pourquoi ils se déséquilibrent pendant la période nocturne pour atteindre des niveaux glycémiques de l'ordre de $4 \mathrm{~g} / \mathrm{L}$. 

COURS DU RAMADAN

Ce problème a fait l'objet d'une littérature relativement abondante $[3,24,28]$ de laquelle il est possible de tirer quelques règles générales.

Chez le diabétique de type 2 non insuliné, le traitement médicamenteux peut être maintenu à son niveau habituel au moins lorsqu'il n'existe aucun risque d'hypoglycémie iatrogène : metformine [29], inhibiteurs de la DPP-4 [30], agonistes des récepteurs du GLP-1 [30,31], inhibiteurs des alpha glucosidases [32] et pour le futur, inhibiteurs du SGLT2 [33] (tableau I).

Pour cette dernière classe de médicaments, la prudence d'utilisation ne porte pas sur le risque hypoglycémique, mais sur le danger de déshydratation. En effet, les inhibiteurs du SGLT2 entrainent une perte d'eau et de sodium avec risque d'hypovolémie [34,35]. Ainsi les inhibiteurs du SGLT2 devraient être fortement déconseillés au cours du Ramadan chez tous les diabétiques vivant dans les pays très chauds lorsque sa période coïncide avec une saison trop chaude.

Par ailleurs, la prudence devrait être la règle lorsque le patient diabétique de type 2 est traité par des antidiabétiques oraux susceptibles d'entrainer des hypoglycémies comme les sulfonylurées et les glinides. Bien que certains auteurs aient proposé d'interrompre ces médicaments pendant le Ramadan, les hypoglycémies induites par les sulfonylurées et les glinides ne sont pas suffisamment fréquentes pour conduire à leur arrêt. Certains proposent de remplacer les sulfonylurées par les glinides, qui comportent moins de risque hypoglycémique [36]. Le mieux est de titrer la dose de ces médicaments à un niveau qui permet d'éviter les hypoglycémies en utilisant la glycémie de fin d'après midi, avant le repas de rupture du jeûne. En effet, cette glycémie est la plus basse de la journée chez tous les diabétiques de type 2 [37] et plus particulièrement chez ceux qui suivent le Ramadan (figures 1 et 3) [12,20]. Dans ces conditions, la dose de sulfonylurée ou de glinide devrait être ajustée de telle manière que ce nadir glycémique de fin d'après midi, dit « de sécurité », reste supérieur à $0,70-0,80 \mathrm{~g} / \mathrm{L}$. 
Chez les diabétiques insulinés, qu'ils soient de type 1 ou 2, le mieux est de conseiller un schéma insulinique de type Basal-Bolus ou Basal-Plus quand ils sont insuffisamment équilibrés avant le Ramadan (Tableau II). Les injections d'analogues rapides doivent être réalisées avant les prises alimentaires qui comportent une charge glucidique significative en qualité et quantité [26]. Pour la pratique, nous considèrerons que tout apport glucidique de plus de 40 à 50 grammes, surtout s'il s'agit de glucides à index glycémique élevé, devrait être accompagné par une injection d'analogue rapide selon les modalités suivantes : 1 unité d'analogue rapide pour 10 grammes d'hydrates de carbone. Pour le repas avant l'aube, cette dose devrait être portée à 1,5 unités pour 10 grammes d'hydrates de carbone, car c'est en fin de nuit que l'insulinorésistance et la production hépatique de glucose sont à leur maximum [38] avec pour conséquences un phénomène de l'aube (remontée glycémique en fin de nuit même en dehors de toute prise glucidique nocturne) et un phénomène de l'aube étendu [39-41]. Ce dernier se traduit par une excursion marquée de la glycémie postprandiale en milieu de matinée.

Au cours du Ramadan, la prise d'un repas avant l'aube, relativement riche en glucides, ne peut que contribuer à majorer l'hyperglycémie de fin de nuit et à rendre la chute glycémique plus aléatoire pendant le reste de la journée. C'est pour cette raison que la règle de 1,5 unités d'analogue rapide de l'insuline pour 10 grammes de glucides devrait être appliquée au repas pris avant l'aube plutôt que la règle générale de 1 unité pour 10 grammes pour les autres repas.

A partir de ces considérations, prenons l'exemple simple d'un patient diabétique de type 2 moyennement équilibré, insuliné depuis plusieurs mois ou années avec un schéma de type basal : une injection d'analogue lent de l'insuline (0,5 unités/kg de poids/jour) avant le dîner. S'il souhaite pratiquer le jeûne religieux du Ramadan, il est préférable de lui proposer de passer à un schéma Basal-Plus ou Basal-Bolus, après avoir réduit la dose d'insuline basale de 10 à $20 \%$ (dans le cas présent passage à 0,4 unité/kg/jour). Les bolus d'analogues rapides de l'insuline doivent être injectés au moment de la rupture du jeûne, du dîner qui suit le repas de rupture du jeûne et du repas pris avant l'aube. Supposons que ces 3 repas (figure 6) apportent respectivement 90,100 et 80 grammes de glucides, ils devraient d'après notre calcul être précédés par l'injection de doses d'analogue rapide égales à 9,10 et 12 unités (figure 6). 
Ces doses sont évidemment à ajuster en fonction des résultats de l'autosurveillance glycémique, qui doit être renforcée dans les jours qui précèdent le Ramadan et poursuivie pendant toute sa durée. De plus, les schémas BasalPlus ou Basal-Bolus doivent être instaurés quelques jours avant le début du Ramadan pour tester la réponse du sujet.

Tout ceci indique que la gestion de l'insulinothérapie au cours du Ramadan n'est pas un problème simple. Dans de nombreux cas, la meilleure attitude serait d'essayer de dissuader tout diabétique insuliné de suivre le jeûne religieux si certaines conditions ne sont pas strictement remplies : observance suffisante d'un programme nutritionnel structuré, acceptation et compréhension d'un schéma insulinique intensifié et augmentation du nombre de tests d'autosurveillance glycémique. 
CONCLUSIONS

Au terme de cette revue, qui est loin d'être exhaustive, mais il est impossible de l'être en raison de l'hétérogénéité des pratiques alimentaires du Ramadan, il apparaît que chez les sujets diabétiques, une attention particulière devrait être donnée aux recommandations alimentaires, avec une vigilance particulière sur les apports caloriques et glucidiques.

Si le médecin considère que les « entorses » ou les " dérapages » nutritionnels ne peuvent être maîtrisés chez un patient diabétique donné, la bonne attitude est de lui déconseiller de pratiquer le jeûne religieux. Ceci est particulièrement évident chez les diabétiques insuffisamment équilibrés à l'état de base, "vulnérables», traités par des médicaments susceptibles d'entraîner des hypoglycémies (sulfonylurées et/ou insuline), et/ou ayant des variations glycémiques importantes entre pics et nadirs. Chez les autres, le jeûne religieux reste possible.

Certains de ces points avaient été formulés lors de la conférence du consensus de Casablanca en 1995 [11], mais l'exemption du jeûne religieux n'avait peut être pas été suffisamment préconisée pour les groupes de diabétiques, où sa pratique risque de s'avérer réellement dangereuse : accident de décompensation aiguë sur le moment et risque de complications diabétiques dégénératives sur le long terme. N'oublions pas que l'organisme d'un diabétique garde la mémoire des épisodes hyperglycémiques et que ces derniers ont un effet cumulatif [19]. A titre d'exemple, une protéine collagénique de paroi artérielle soumise à la formation de produits avancés de la glycation persiste pendant plusieurs années, jusqu'à son élimination au moment de son renouvellement naturel [17].

Les auteurs déclarent n'avoir aucun conflit d'intérêt avec le contenu de cet article. 


\section{Les points essentiels}

- La pratique du Ramadan chez le patient diabétique demande une attention particulière

- Un excès d'apports caloriques et glucidiques est souvent observé avec pour conséquences une prise pondérale, des montées glycémiques anormales après le repas de rupture du jeûne et une hyperglycémie nocturne soutenue.

- Chez les patients diabétiques insulinés, les schémas insuliniques intensifiés couplés à une autosurveillance glycémique codifiée sont fortement recommandés

- Quel que soit le type de diabète, pendant la période du Ramadan, le patient devrait suivre un programme nutritionnel structuré, préparé en amont du mois de jeûne.

- Le déséquilibre hyperglycémique du Ramadan peut avoir des conséquences sur le long terme s'il se répète tous les ans pendant plusieurs années en raison de l'effet mémoire des périodes d'hyperglycémie.

- L'exemption du jeûne religieux devrait être sérieusement envisagée chez les sujets ayant un équilibre glycémique insuffisant en dehors du Ramadan, chez les femmes diabétiques enceintes ou présentant un diabète gestationnel, chez tous les sujets vulnérables et chez toutes les personnes diabétiques qui ne peuvent pas ou ne souhaitent pas adapter leur traitement diététique et pharmacologique aux modifications du mode de vie engendrées par le Ramadan.

- Une personne diabétique n'observant pas le jeûne peut néanmoins être conduite à certaines modifications alimentaires significatives, suite aux changements de rythmes alimentaires adoptés par son groupe social ou par son entourage. 


\section{Références bibliographiques}

[1] Diabetes fact sheet. Available from :

\section{http://www.who.int/mediacentre/factsheets/fs312/en/}

[2] Salti I, Bénard E, Detournay B et al. EPIDIAR study group. A population-based study of diabetes and its characteristics during the fasting month of Ramadan in 13 countries : results of the epidemiology of diabetes and Ramadan 1422/2001 (EPIDIAR) study. Diabetes Care 2004 ;2306-2311

[3] Al-Abouj M, Assaad-Khalil S, Buse $\mathrm{J}$ et al. Recommendations for management of diabetes during Ramadan. Update 2010. Diabetes Care 2010 ;33:18951902

[4] Zimmet P, Alberti KGMM, Shaw J. Global and societal implications of the diabetes epidemic. Nature $2001 ; 414: 782-787$

[5] Fasting in religion. Available from :

http://altreligion.about.com/od/ritualsandpractices/a/Fasting-In-Religion.htm

[6] Trepanowski JF, Bloomer RJ. The impact of religious fasting on human health. Nutr J $2010 ; 9: 57-65$.

[7] Sadeghirad B, Motaghipisheh S, Kolahdooz F et al. Islamic fasting and weight loss : a systematic review and meta-analysis. Public Health Nutr $2012 ; 17: 396-406$

[8] Colette C, Monnier L. Diététique des états diabétiques. In : Diabétologie (2 ${ }^{\text {ème }}$ édition). L Monnier Ed. Elsevier Masson. Issy-les-Moulineaux. 2014 ;91-107

[9] Havel RJ. Caloric homeostasis and disorders of fuel transport. N Engl J Med $1972 ; 287: 1186-1192$

[10] DeFronzo RA. Pathogenesis of type 2 diabetes :metabolic and molecular implications for identifying diabetes genes. Diabetes Rev $1997 ; 5: 177-269$

[11] International meeting on Diabetes and Ramadan Recommendations : Edition of the Hassan II Foundation for Scientific and Medical Research on Ramadan. Casablanca, Morocco, FRSMR, 1995

[12] Lessan N, Hannoun Z, Hasan H et al. Glucose excursions and glycaemic control during Ramadan fasting in diabetic patients : Insights from continuous glucose monitoring (CGM). Diabetes Metab 2015 ; 41:36 
[13] Monnier L, Wojtusciszyn A, Colette C et al. The contribution of glucose variability to asymptomatic hypoglycemia in persons with type 2 diabetes. Diabetes Technol Ther $2011 ; 13: 813-818$

[14] Ezzat S, Amin M. Energy intake, dietary pattern and physical activity during the holy month of Ramadan and their impact on body weight. Int J Food Nutr Public Health. 2013 ; 6: 291-309

[15] Bakhotmah BA. The puzzle of self-reported weight gain in a month of fasting (Ramadan) among a cohort of Saudi families in Jeddah, Western Saudi Arabia. Nutr J $2011 ; 10: 84 .-91$

[16] Sachs DB. Measurement of hemoglobin A1c. A new twist on the path to harmony. Diabetes Care $2012 ; 35: 2674-2680$

[17] Lyons TJ, Jenkins AJ. Glycation, oxidation and lipoxidation in the development of the complications of diabetes : a carbonyl stress hypothesis. Diabetes Rev $1997 ; 5: 365-391$

[18] Brownlee M. Biochemistry and molecular cell biology of diabetic complications. Nature $2001 ; 414: 813-820$

[19] Holman RR, Paul SK, Bethel MA et al. 10-year follow-up of intensive glucose control in type 2 diabetes. N Engl J Med 2008 ;359:1577-1589

[20] Monnier L, Bonnet F, Colette C. New insights on glucose homeostasis during Ramadan. Diabetes Metab $2015 ; 41: 1-4$

[21] Dinneen S, Gerich JE, Rizza R. Carbohydrate metabolism in non-insulin dependent diabetes mellitus.N Engl J Med $1992 ; 327:$ :707-713

[22] Monnier L. Is postprandial glucose a neglected cardiovascular risk factor in type 2 diabetes ? Eur J Clin Invest $2000 ; 30$ (suppl 2) :3-11

[23] Cobelli C, Mari A, Ferrannini E. Non-steady state : error analysis of Steele's model and development for glucose kinetics. Am J Physiol (Endocrinol Metab) 1987 ;252 :E679-E689

[24] Kausbal K, Raina SK. Risk approach strategy in diabetics on fasting evidence base through narrative review with a special focus on Ramadan fasting. J Soc Health Diabetes. $2015 ; 3: 11-15$

[25] Alkaabi JM, Al-Dabbagh B, Ahmad S et al. Glycemic indices of five varieties of dates in healthy and diabetic subjects. Nutr J $2011 ; 10: 59$ 
[26] Foster-Powell K, Holt SHA, Brand-Miller JC. International table of glycaemic index and glycaemic load value 2002. Am J Clin Nutr $2002 ; 76$ :5-56

[27] Heymsfield SB, Harp JB, Reitman ML et al. Why do obese patients not lose more weight when treated with low-caloric diets ? A mechanistic perspective. Am J Clin Nutr 2007 ;85:346-354

[28] El-Aziz B, Skalli S, Chadli A et al. Diabète et Ramadan : mise au point sur les recommandations récentes. Méd mal Métabol $2014 ; 8: 221-225$

[29] Cusi K, DeFronzo RA. Metformin : a review of its metabolic effects. Diabetes Rev $1998 ; 6: 89-131$

[30] Drucker DJ, Nauck MA. The incretin system glucagon-like-peptide-1 receptor agonists and dipeptidyl-peptidase inhibitors in type 2 diabetes. Lancet $2006 ; 368: 1696-1705$

[31] Owens DR, Monnier L, Bolli GB. Differential effects of GLP-1 receptor agonists on components of dysglycemia in individuals with type 2 diabetes mellitus.

Diabetes Metab $2013 ; 39: 485-496$

[32] Lebovitz HE. Alphaglucosidase inhibitors as agents in the treatment of diabetes. Diabetes Rev 1998 ; 6:132-145

[33] Bailey CJ..Renal glucose reabsorption inhibitors to treat diabetes. Trends Pharmacol Sci $2011 ; 32$ :63-71

[34] Gourdy P. Effets indésirables et sécurité d'emploi des inhibiteurs des SGLT2. Méd mal Métabol 2015 ;9 (suppl. 1) :S41-\$48

[35] Halimi S, Vergès B. Adverse effects and safety of SGLT2 inhibitors. Diabetes Metab $2014 ; 40$ :S28-S34

[36] Lebovitz HE. Insulin secretagogues : old and new. Diabetes Rev $1999 ; 7$ :139153

[37] Monnier L, Colette C, Dunseath G et al. The loss of postprandial glycemic control precedes stepwise deterioration of fasting with worsening diabetes.

Diabetes Care 2007 ;30:263-269

[38] Boden G, Chen X, Urbain JL. Evidence for a circadian rythm of insulin sensitivity in patients with NIDDM caused by cyclic changes in hepatic glucose production. Diabetes 1996 ; 1044-1050 
[39] Monnier L, Colette C, Sardinoux M et al. Frequency and severity of the dawn phenomenon in type 2 diabetes : relationship to age. Diabetes Care $2012 ; 35: 2597-2599$

[40] Monnier L, Colette C, Dejager S et al. Magnitude of the dawn phenomenon and its impact on the overall glucose exposure in type 2 diabetes. Is this of concern ? Diabetes Care $2013 ; 36: 4057-4062$

[41] Monnier L, Colette C, Dejager S et al. The dawn phenomenon in type 2 diabetes. How to assess it in clinical practice ? Diabetes Metab 2015 ; 41 :132137 


\section{Légendes des figures}

Figure 1 : Profils glycémiques sur 24 heures au cours du Ramadan chez des sujets témoins non diabétiques et chez des patients diabétiques. En rouge : tous les diabétiques ( $n=56)$, quel que soit leur traitement. En vert : les diabétiques $(n=6)$ traités par insuline \pm antidiabétiques oraux [d'après la référence 12].

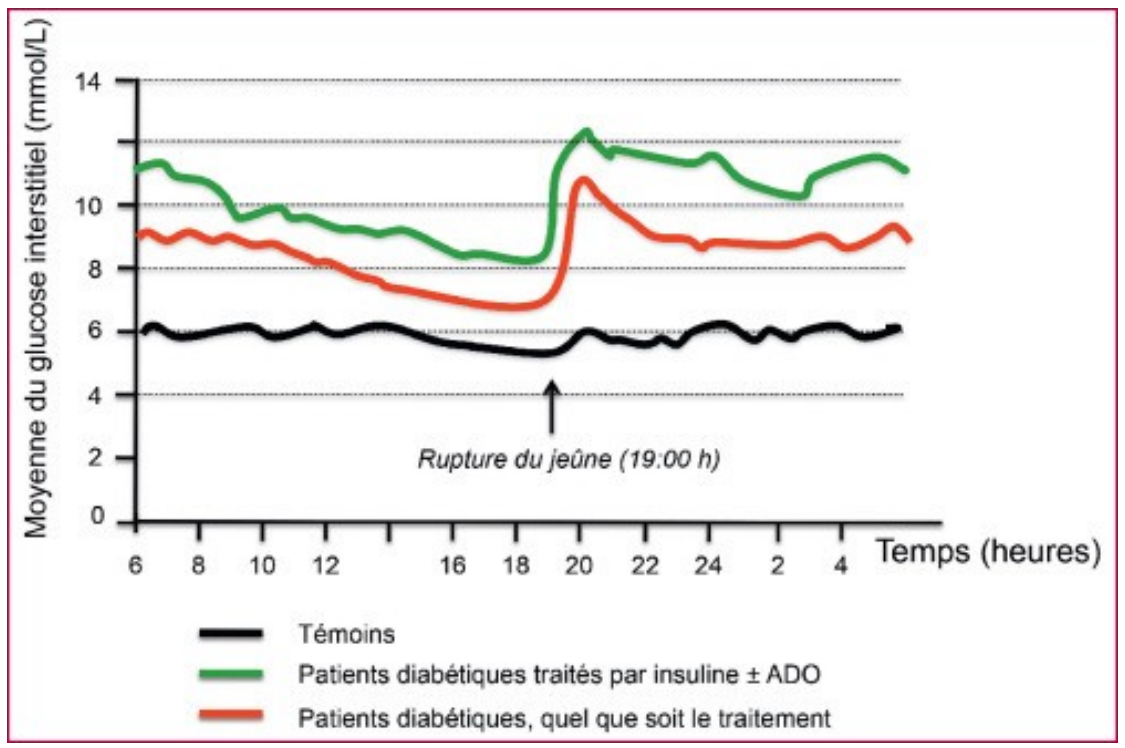

Figure 2 : Profils glycémiques sur 24 heures chez des patients diabétiques explorés au cours et en dehors du Ramadan [d'après la référence 12].

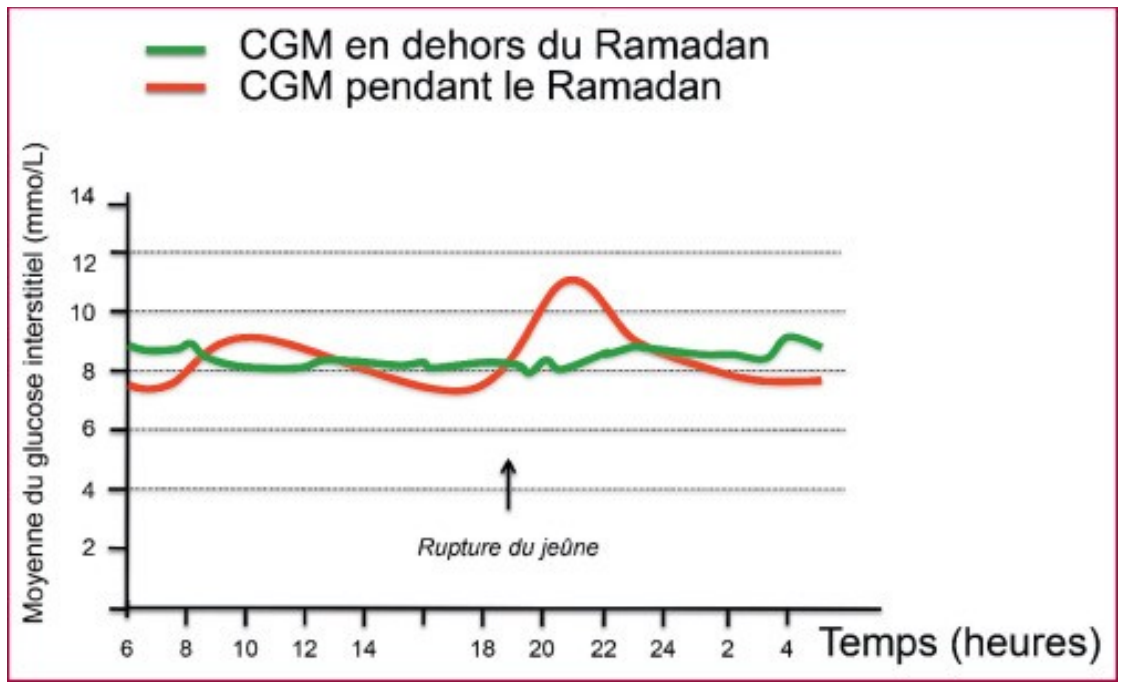


Figure 3 : Profils glycémiques sur 24 heures chez un patient exploré au cours du Ramadan. Les flèches rouges verticales indiquent les moments des repas de rupture du jeûne. Les autres prises alimentaires entre le coucher et le lever du soleil ne sont pas indiquées car non enregistrées par le patient [d'après la référence 20].

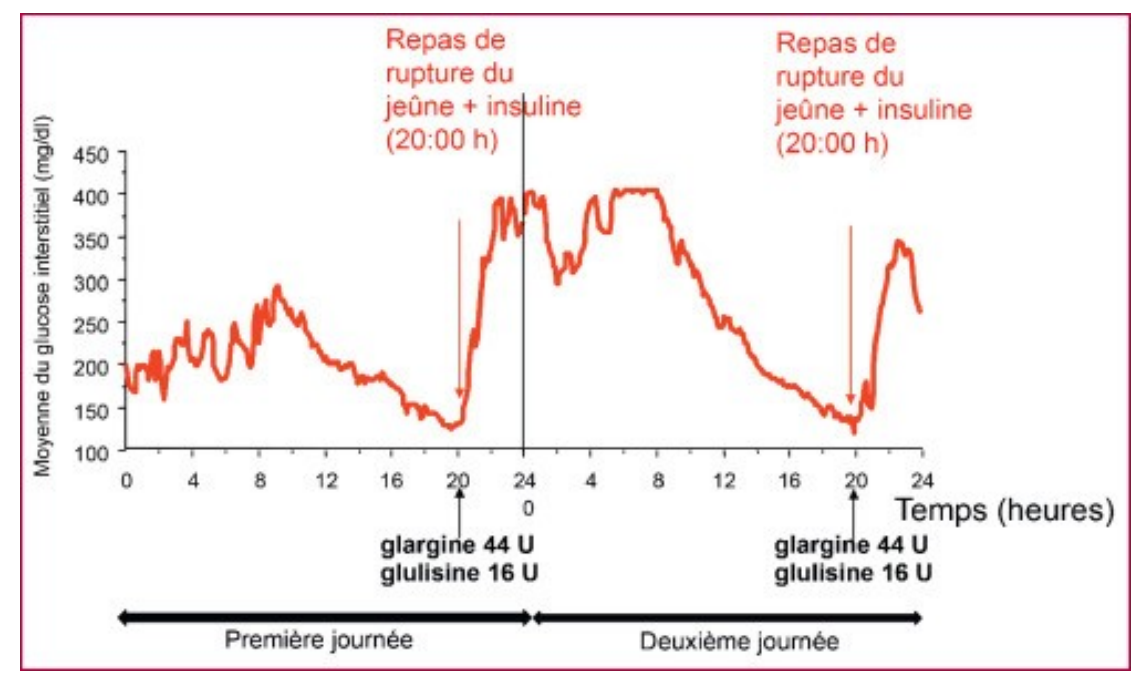

Figure 4 : Assortiment de plats traditionnels Marocains proposés au cours du repas de rupture du jeûne.

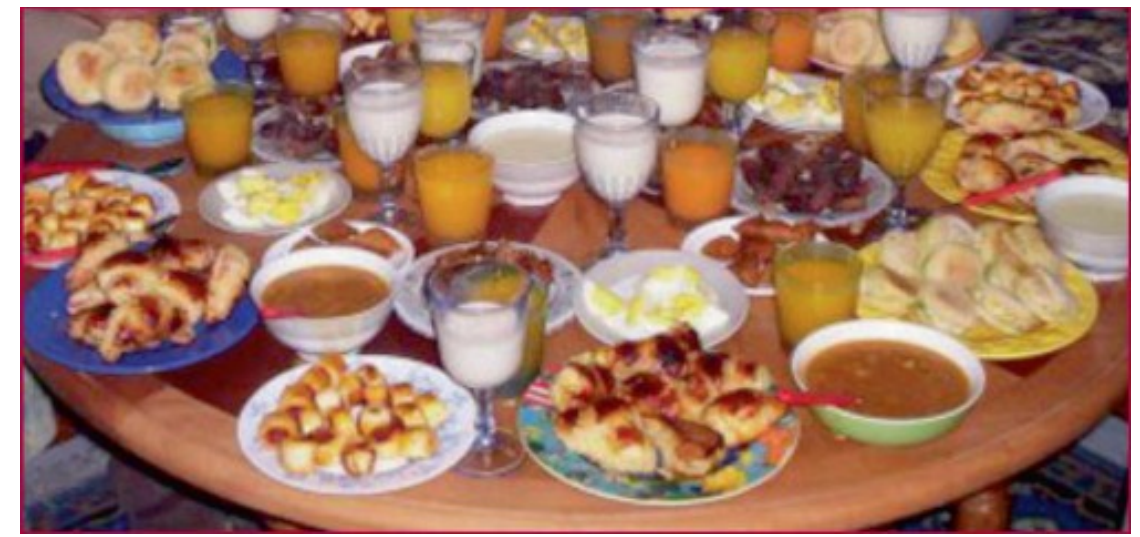


Figure 5 : Répartition schématique des apports glucidiques entre le coucher et le lever du soleil. Cette répartition n'est qu'une proposition en sachant que le repas de rupture du jeûne (Iftar) et le repas pris avant l'aube (Sohour) sont quasi constants, la variable étant constituée d'une part par le dîner qui suit de 2 ou 3 heures l'Iftar et d'autre part par les collations nocturnes.

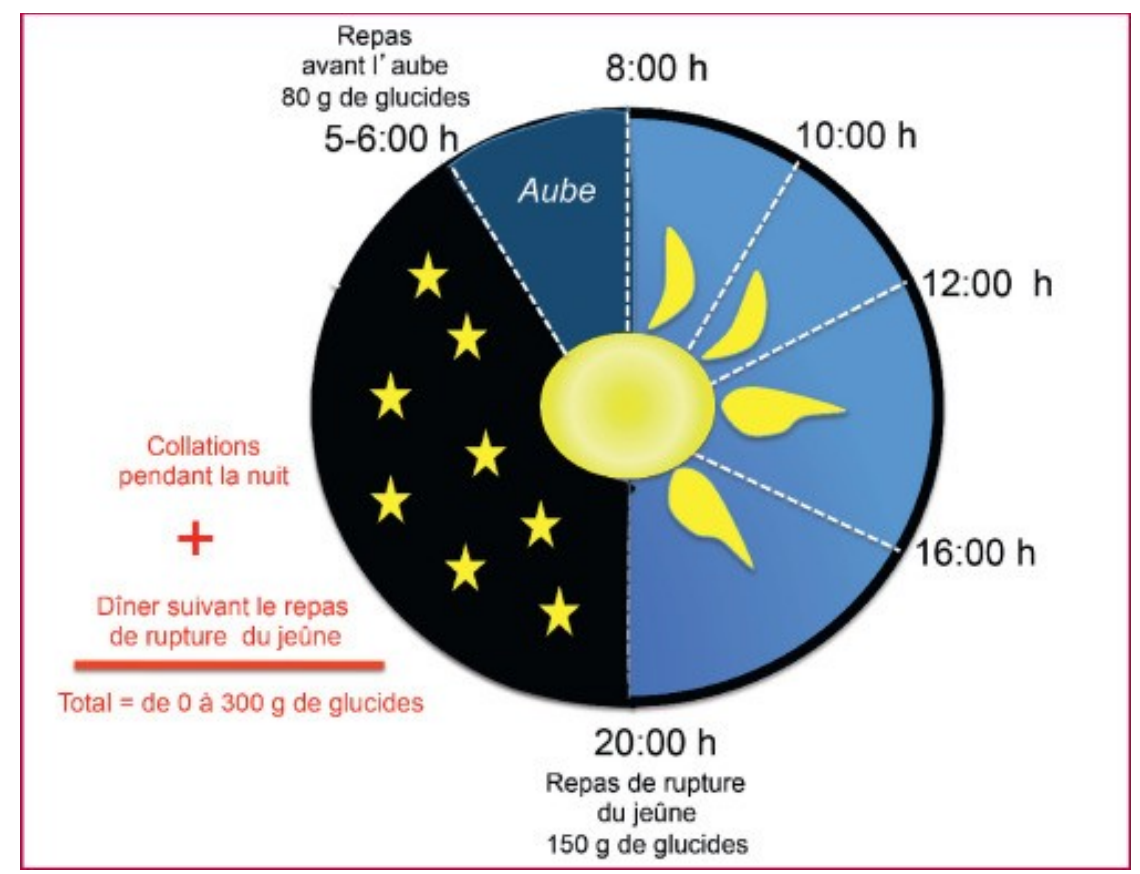

Figure 6 : Répartition suggérée pour les bolus d'analogues rapides de l'insuline au cours du Ramadan chez un diabétique insuliné, sous schéma Basal-Bolus et prenant 3 repas pendant la nuit avec les apports glucidiques suivants : $90 \mathrm{~g}$ au repas de rupture du jeûne (Iftar), $100 \mathrm{~g}$ au dîner qui suit et $80 \mathrm{~g}$ au repas pris avant l'aube (Sohour).

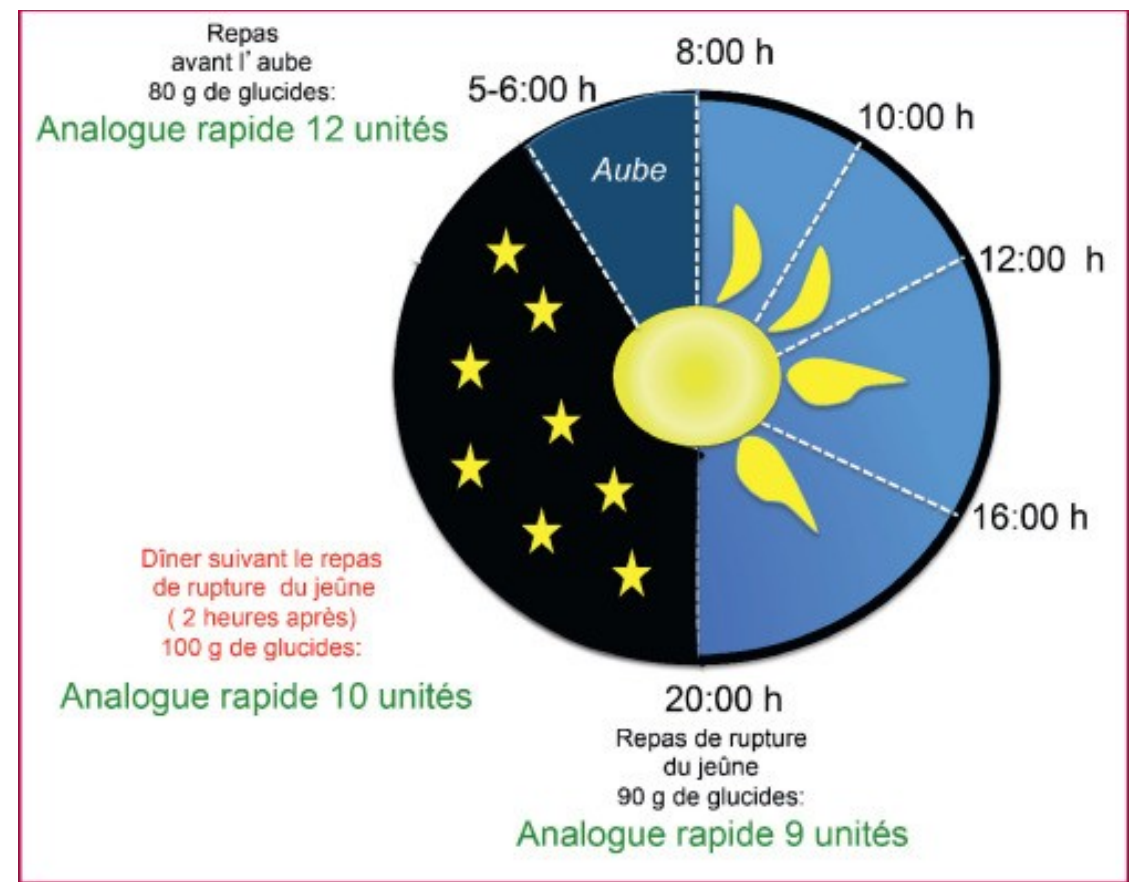


Tableau I : Utilisation des antidiabétiques pendant le Ramadan. Risques et recommandations pratiques.

Tableau I.

Utilisation des traitements antidiabétiques pendant le Ramadan : risques et recommandations pratiques.

\begin{tabular}{|l|c|l|}
\hline $\begin{array}{l}\text { Antidiabétique } \\
\text { Metformine }\end{array}$ & $\begin{array}{c}\text { Risque } \\
\text { Non }\end{array}$ & $\begin{array}{l}\text { En pratique } \\
\text { Doses inchangées }\end{array}$ \\
\hline $\begin{array}{l}\text { Inhibiteurs des } \\
\text { a-glucosidases }\end{array}$ & Non & Doses inchangées \\
\hline Inhibiteurs de la DPP-4 & Non & Doses inchangées \\
\hline $\begin{array}{l}\text { Agonistes des récepteurs } \\
\text { du GLP-1 }\end{array}$ & Non & Doses inchangées \\
\hline \multicolumn{1}{|c|}{ Sulfamides } & Hypoglycémies & $\begin{array}{l}\text { Diminuer la dose en titrant sur la glycémie la plus basse, } \\
\text { celle de fin d'après-midi (nadir) }\end{array}$ \\
\hline Glinides & Hypoglycémies & Diminuer la dose et répartir \\
\hline sur la nuit & & \\
\hline Inhibiteurs du SGLT-2 & Déshydratation & À ne pas prescrire, ou à arrêter \\
\hline
\end{tabular}

DPP-4 : dipeptidylpeptidase IV ; GLP-1 : glucagon-like peptide-1 ; SGLT-2 : co-transporteur sodium-glucose de type 2 . 
Tableau II : Utilisation et modalités de l'insulinothérapie pendant le Ramadan.

Risques et recommandations pratiques.

Tableau II.

Utilisation et modalités de l'insulinothérapie pendant le Ramadan : risques et recommandations pratiques.

\begin{tabular}{|c|c|c|}
\hline Insuline & Risque & En pratique \\
\hline \multirow{2}{*}{$\begin{array}{l}\text { DT2 } \\
\text { insulino- } \\
\text { traité }\end{array}$} & \multirow[t]{2}{*}{$\begin{array}{l}\text { Hypoglycémies et } \\
\text { hyperglycémies }\end{array}$} & $\begin{array}{l}\text { - Opter pour une réduction de l'insuline basale }(-20 \% \\
\text { d'insuline lente ou semi-lente). }\end{array}$ \\
\hline & & $\begin{array}{l}\text { - Introduire des bolus d'insuline prandiale au moment des } \\
\text { repas nocturnes comportant une prise glucidique consistante } \\
\text { (voir texte). }\end{array}$ \\
\hline \multirow[t]{2}{*}{ DT1 } & $\begin{array}{l}\text { - Hypoglycémies et } \\
\text { hyperglycémies }\end{array}$ & - Opter pour une réduction de l'insuline basale $(-20 \%)$. \\
\hline & - Cétoacidose & $\begin{array}{l}\text { - Répartir les bolus d'insuline prandiale selon les prises } \\
\text { alimentaires (voir texte). }\end{array}$ \\
\hline
\end{tabular}

DT2 : diabète de type 2 ; DT1 : diabète de type 1. 